\section{Subjective visual perceptions during vitreoretinal surgery under local anaesthesia}

SB Vohra', C Anya², T Farooq ${ }^{2}$ and PI Murray ${ }^{1,2}$

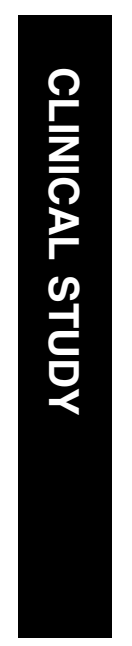

reassured that they are not normally frightening. Eye (2009) 23, 1831-1835; doi:10.1038/eye.2008.325; published online 24 October 2008

Keywords: subjective visual perceptions; vitreoretinal surgery; local anaesthesia; sub-Tenon's blocks

\section{Introduction}

Patients undergoing intraocular surgery under local anaesthesia (LA) frequently perceive light and other visual experiences during the procedure, such as seeing colours, movements, and instruments. Although the scale of visual perceptions is well documented during cataract surgery, ${ }^{1-6}$ the extent of these experiences during vitreoretinal surgery is less well known. ${ }^{7,8}$ As vitreoretinal procedures are increasingly being performed under LA, ${ }^{9}$ and appear well tolerated by the patient, ${ }^{10}$ it is vital that we adequately and appropriately inform patients of what they might experience during the surgery.

We undertook a prospective questionnaire study of patients undergoing vitreoretinal procedures under LA to document their subjective visual perceptions during the surgery, and to look at any variables that may have an effect on these perceptions.

\section{Patients and methods}

A prospective questionnaire-based study was designed. One part of the questionnaire included demographic data, preoperative visual acuity, indications for surgery, coexisting ocular pathology, previous ocular surgery, duration and details of the operative procedure, details of the LA block including volume and
${ }^{1}$ Birmingham and Midland Eye Centre, City Hospital, Birmingham, UK

${ }^{2}$ Academic Unit of Ophthalmology, University of Birmingham, Birmingham, UK

Correspondence: PI Murray, Academic Unit of Ophthalmology, University of Birmingham, Birmingham and Midland Eye Centre,

City Hospital,

Sandwell and West Birmingham Hospitals NHS Trust,

Dudley Road,

Birmingham B18 7QU, UK

Tel: + 01215076851 ;

Fax: +0121507 6853 .

E-mail: p.i.murray@

Received: 5 May 2008 Accepted in revised form: 26 September 2008 Published online: 24 October 2008 a poster at the 2007 RCOphth Annual Congress, Birmingham and at the 2nd World Congress of Ophthalmic Anaesthesia, 2008, Cairo, Egypt bham.ac.uk

This study was presented as 
concentration and use of hyaluronidase, efficacy of the blocks, and any supplementary ocular injections or the use of sedation. The other part of the questionnaire recorded each patient's experiences of visual perceptions with the operated eye through the duration of the surgical procedure.

We prospectively recruited a total of 80 patients undergoing a variety of vitreoretinal procedures under LA. All patients were interviewed within $30 \mathrm{~min}$ of completion of surgery. Patients with all types of ocular pathology and levels of visual acuity were included, but those with the history of dementia or loss of insight into the process were excluded. No patient refused to be part of the survey. All patients were documented to have a least perception of light acuity in the operated eye before surgery.

Patients were asked whether they saw complete darkness or light during the surgery and whether they perceived any changes in the light intensity throughout the procedure. Those who saw light during the surgery were further questioned about visual perception of flashes, colours, movements, and seeing instruments. They were also asked whether they had been warned in the preoperative period regarding the visual perceptions they might experience, and, if not, whether they would have liked to have been warned. Finally, patients were asked about any pain during the procedure, and whether they had found the visual experiences frightening.

\section{Statistical analysis}

Statistical analysis was carried out using SPSS 15.0 (SPSS Inc., Chicago, IL, USA). Pair-wise association of categorical variables was examined using a $\chi^{2}$-test. In one case, Fisher's exact test was used due to small cell frequencies. A two-sample $t$-test was used to compare mean ages between the groups of patients.

Logistic regression analysis was undertaken to quantify any association between individual factors for visual perceptions (light, movement, flashes, colours, and instruments) after adjusting for other variables (such as age, gender, type of procedure, diagnosis, preoperative acuity, duration of surgery, type of block, use of sedation, and degree of akinesia).

\section{Results}

There were 48 men and 32 women, with the majority of patients $(49 / 80,61.3 \%)$ aged 65 years or above. A total of 72 patients underwent pars plana vitrectomy as the primary procedure with eight patients having removal of silicone oil (ROSO), including three combined with phakoemulsification cataract extraction. Indications for vitrectomy were retinal detachment $(n=35)$, vitreous haemorrhage $(n=15)$, macular hole $(n=8)$, epiretinal membrane peel $(n=3)$, dropped nucleus $(n=2)$, and other $(n=9)$. Preoperative visual acuity was $6 / 60$ or worse in 60 eyes (including $\mathrm{CF} n=4, \mathrm{HM} n=17$, and PL $n=3)$, and better than $6 / 60$ in 20 eyes $(6 / 5-6 / 12(n=11)$, $6 / 18-6 / 36(n=9))$.

A sub-Tenon's block was undertaken in 62/80 (77.5\%) patients, the majority through a single inferonasal approach, and a peribulbar block in the remainder with a single medial canthal injection using a 25-mm 25G needle. The commonest anaesthetic agent used was $0.75 \%$ levobupivacaine in $62(77.5 \%)$ of patients, with hyaluronidase added to the majority of blocks (73/80, 91.3\%). A 'top-up' supplementary injection was required in $10 / 80(12.5 \%)$ patients (six following a sub-Tenon's block and two following a peribulbar block). Of the 10 top ups, two were performed preoperatively and eight intraoperatively. One-fifth of the patients $(16 / 80,20 \%)$ received awake sedation. Of these, 10 patients received a small intravenous bolus of midazolam just before surgery and six in the intraoperative period.

An extraocular muscle movement (akinesia score) was noted at $5 \mathrm{~min}$ after injection of the LA. Patients were asked to look in the four directions of gaze (up, down, right, and left). The movement of the globe was compared with the unblocked eye. For each direction, a score of ' 0 ' was assigned to total akinesia, a score of ' 1 ' denoted a flicker, whereas ' 2 ' and ' 3 ' denoted a moderate and full movements, respevtively. The individual muscle movement scores were added. Thus, a total score of 12 implied full movement of the globe (ie, complete block failure) ${ }^{11}$ The akinesia scores were only recorded in 76 patients. Of these, total akinesia (ie, score 0) was achieved in $40(52.6 \%)$ patients. A score of 1-4 was achieved in 24 (31.6\%) patients, and $12(15.8 \%)$ patients scored 5 or greater.

Over half $(45 / 80,56.3 \%)$ of the surgical procedures were completed within $1 \mathrm{~h}, 31 / 80(38.7 \%)$ procedures took $1-2 \mathrm{~h}$, and $4 / 80$ procedures $(5 \%)$ were completed in 2-3h.

\section{Details of visual perceptions}

A total of $57 / 80(71.3 \%)$ patients perceived light at the onset of their surgery with the remainder $(n=23)$ admitting to complete darkness (NPL). Of these 23 patients, 15 later perceived light during the procedure. The 72 patients that saw light at some time during their surgery experienced the following perceptions:

\section{Flashing lights}

Flashing lights were seen by $24(33.3 \%)$ patients.

\section{Colours}

Seeing colours was reported by $45(62.5 \%)$ patients. Of these, 19 patients saw only one colour and 26 saw two or 
more colours. Seeing a 'rainbow' was perceived by seven $(9.7 \%)$ patients. Table 1 illustrates the range of colours perceived.

\section{Movements}

Some kind of movement was observed by $51 / 72$ (70.83\%) patients. Although most patients found these rather nondescript, nine saw multicolour swirls, such as oil floating on water, two saw 'strings', and three reported seeing tadpole-like structures and perceived zigzag amoebatype movements, with black dots seen by one patient.

\section{Instruments}

Seeing some form of instrument was reported by 38 $(52.8 \%)$ patients. Various descriptions included visualisation of a 'black pipe with a hole at the end', a 'thin bar' or a 'probe-like' structure approaching from one or other side of the visual field. Others described seeing 'clips', 'curved tweezers', 'crocodile's snout with teeth', 'machete,' and 'needles'. Some patients also reported seeing a 'probe with a light at the end'. A few described seeing 'wispy filaments' being lifted from inside of their eye with these instruments. One patient drew a picture of 'clips,' which he described as 'two crocodiles fighting', and another drew a sketch of a 'beaten tin plate' (Figure 1a and b).

\section{Relationship of visual perceptions and the density of the block}

In the 40 patients where total akinesia of the globe was achieved at the outset (akinesia score $=0), 24(60 \%)$ saw movements, $23(57.5 \%)$ saw colours, and 18 (45\%) saw instruments. Of the 24 patients with near total akinesia (scores of 1-4), 14 (58.3\%) saw movements, 11 (45.8\%) saw colours, and $10(41.7 \%)$ saw instruments. Patients with scores of 5 or greater $(n=12)$, eight $(66.7 \%)$ saw movements, six (50\%) saw colours, and eight (66.7\%) saw instruments.

Table 1 Colours perceived by 72 patients

\begin{tabular}{lc}
\hline Colour & Number $(\%)^{\mathrm{a}}$ \\
\hline Red & $19(42.2)$ \\
Blue & $9(20.0)$ \\
Yellow & $8(17.8)$ \\
Green & $7(15.6)$ \\
Orange & $6(13.3)$ \\
Purple & $3(6.7)$ \\
Brown & $1(2.2)$ \\
\hline
\end{tabular}

aSome patients saw more than one colour.

\section{Patient acceptance of the procedure}

The majority of patients $(69 / 80,86.25 \%)$. experienced no pain during surgery, $9 / 80(11.25 \%)$ felt mild discomfort, and $1 / 80(1.25 \%)$ reported moderate pain. Only one patient described the pain as severe. The visual perceptions were described as 'pleasant' by $16 / 72$ (22.22\%), 'bearable' by 52/72 (72.22\%), and only two patients $(2.7 \%)$ thought they were 'frightening,' although one of these found these perceptions to be pleasant as the surgery progressed. With regards to being warned preoperatively about the possible visual perceptions they might experience during the surgery, only four (5\%) patients would like to have been warned. The majority $(42(52.5 \%))$ did not, and $34(42.5 \%)$ of the patients were ambivalent about it.

\section{Statistical analysis}

Initial linear analysis showed the following association: younger patients perceiving light $(P=0.04)$ and flashes $(P=0.02)$, women seeing instruments as compared with men $(P=0.01)$, patients having a sub-Tenon's block perceiving light $(P=0.02)$, patients undergoing vitrectomy seeing instruments compared with those

\section{a}
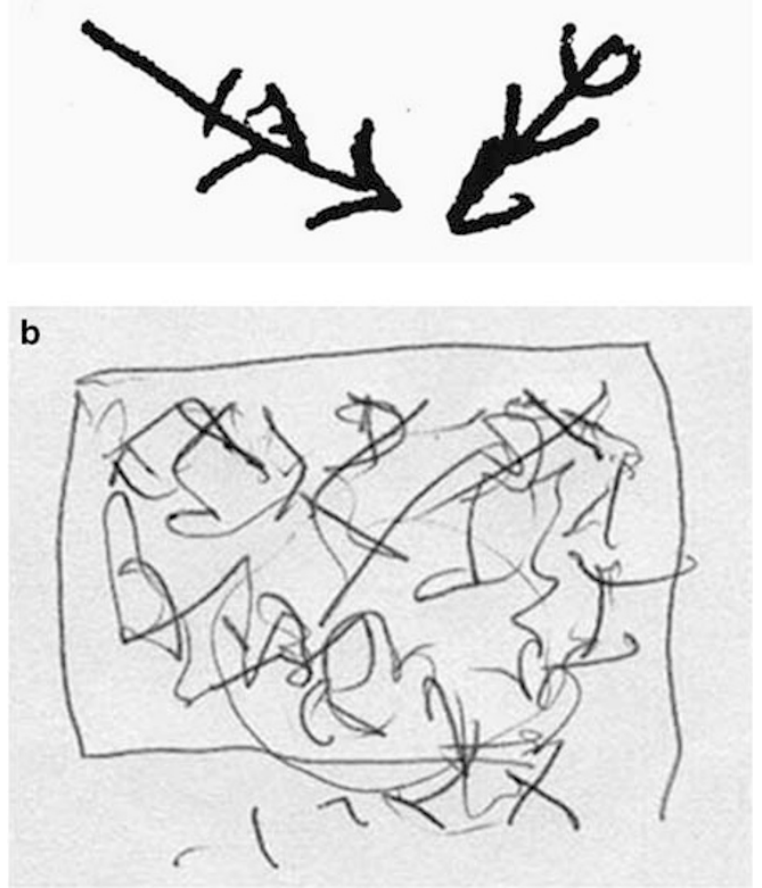

Figure 1 (a) Patient's drawing of two clips described as 'two crocodiles fighting'. (b) A sketch of visual perceptions described as 'beaten tin plate' by another patient. 
Table 2a Statistical analysis of visual perceptions

\begin{tabular}{llllll}
\hline & Light & Movement & Flashes & Colours & Instruments \\
\hline Age & $\mathbf{0 . 0 4}^{*}$ & 0.11 & $\mathbf{0 . 0 2}^{*}$ & 0.08 & 0.27 \\
Gender & 0.58 & 0.06 & 0.89 & 0.72 & $\mathbf{0 . 0 1}$ \\
$\begin{array}{l}\text { Peribulbar/ } \\
\text { Sub-Tenon }\end{array}$ & $\mathbf{0 . 0 2 *}$ & 0.10 & 0.71 & 0.57 & 0.37 \\
VA score & 0.83 & 0.49 & 0.83 & 0.89 & 0.07 \\
Vitrectomy vs & 0.65 & 0.14 & 0.82 & 0.45 & $\mathbf{0 . 0 3}$ \\
ROSO & & & & & \\
Akinesia & 0.20 & 0.46 & 0.90 & 0.22 & 0.69 \\
Awake sedation & 0.59 & $\mathbf{0 . 0 1}^{*}$ & 0.95 & 0.76 & 0.09 \\
\hline
\end{tabular}

$\mathrm{ROSO}=$ removal of silicone oil; VA, visual acuity.

$\chi^{2}$-test of individual associations showing $P$-values of association between the column variable and the row variable except for age where $t$-test was used. The significant values are shown with a ${ }^{*}$ symbol Fisher's exact test was used in one case indicated by ${ }^{+}$due to small cell frequencies.

Table 2b Further analysis of visual perceptions

\begin{tabular}{llcccc}
\hline & Light & Movement & Flashes & Colours & Instruments \\
\hline Age & 0.60 & 0.06 & $\mathbf{0 . 0 3}$ & 0.29 & $\mathbf{0 . 0 4}^{*}$ \\
Gender & 0.68 & 0.14 & 0.41 & 0.48 & 0.05 \\
$\begin{array}{l}\text { Peribulbar/ } \\
\text { Sub-Tenon }\end{array}$ & $\mathbf{0 . 0 0 5}$ & 0.51 & 0.56 & 0.52 & 0.95 \\
VA score & 0.68 & 0.71 & 0.98 & 0.68 & 0.16 \\
Vitrectomy vs & 0.13 & 0.45 & 0.84 & 0.77 & $\mathbf{0 . 0 3}^{*}$ \\
ROSO & & & & & \\
Akinesia & 0.13 & 0.72 & 0.57 & 0.25 & 0.06 \\
Awake sedation & 0.19 & 0.10 & 0.38 & 0.82 & $\mathbf{0 . 0 4}^{*}$ \\
\hline
\end{tabular}

$\mathrm{ROSO}=$ removal of silicone oil; VA, visual acuity.

Logistic regression analysis showing $P$-values of association between the column and individual row variables after adjusting for the other row variables. The significant values are shown with $a{ }^{*}$ symbol.

having $\operatorname{ROSO}(P=0.03)$, and those who did not receive any sedation seeing movements $(P=0.01$; Table $2 \mathrm{a})$.

Logistic regression analysis confirmed many of the linear analysis findings. Younger people were significantly associated with experiencing flashing lights $(P=0.03)$ and also seeing instruments $(P=0.04)$. The other significant associations were patients having a sub-Tenon's block perceiving light $(P=0.005)$, patients undergoing vitrectomy seeing instruments compared with those having ROSO $(P=0.03)$, and those who did not have sedation, seeing instruments $(P=0.04$;

Table 2b).

Logistic regression analysis showed no association between visual perceptions and the gender of the patient, preoperative visual acuity, or the density of the block.

\section{Discussion}

Patients undergoing phakoemulsification cataract extraction under topical or regional anaesthesia have been shown to experience a wide variety of visual sensations during surgery. ${ }^{1-6}$ To date, only two earlier studies have looked at subjective visual perceptions in patients undergoing vitreoretinal surgery under LA. ${ }^{7,8}$ Both studies have shown similar observations to our own. In a series of 65 patients, Tan et $a l^{7}$ found $61.5 \%$ saw colours, $55.4 \%$ movements, and $50.8 \%$ instruments. Sugisaka $e t a l^{8}$ in their series of 101 patients reported figures of $72.3,56.4$, and $53.5 \%$, respectively. Our study showed comparable results with $62.5 \%$ of patients that perceived light during the surgery also saw colours and movements, and $52.8 \%$ patients saw instruments with some accurately describing their shape and type despite having no previous knowledge of them. A similar finding has been reported previously. ${ }^{12}$

Some studies have alluded to these sensations as being frightening. In a multicentre study conducted in Singapore, Hong Kong, and Ireland, 9/65 patients (13.8\%) undergoing vitrectomy were frightened by their visual experiences during surgery. Contributory risk factors were quoted to be a younger age and longer duration of surgery. ${ }^{7}$ The results of our study show that only two patients $(2.7 \%)$ were frightened and one of them later found the perceptions to be pleasant. This is also less than the $5.9 \%$ found by Sugisaka et $a l^{8}$ in Japan. The numbers are too small to conclude whether this could be due to cultural differences.

Only eight $(10 \%)$ of our patients failed to perceive light at any time during the procedure, which is in agreement with Sugisaka et al, although Tan et $a l^{7}$ found a quarter of their patients failed to perceive light. It is difficult to explain the higher figure in the latter study, but is unlikely to be due to the volume of the LA. Tan et al used 5-9 $\mathrm{ml} \mathrm{LA}$, but Sugisaka et $\mathrm{al}^{8}$ reported that the amount of LA was not found to be statistically significant in relation to the visual sensations experienced. Both these studies did not allude to the density or the efficacy of the block. We measured the degree of the motor blockade following the local block as this has traditionally been used to gauge the degree of sensory block. In our study, there was no difference in the numbers of patients seeing colours, movements, and instruments, if they had a dense block (total akinesia), or had slight or moderate movement of the eye. Throughout the whole range of 'akinesia' scores, the various visual perceptions appeared equally distributed. There also appeared no relation to the type of anaesthetic agent used. Tan et $a l^{7}$ used a mixture of $2 \%$ lignocaine and $0.75 \%$ bupivacaine, Sugisaka $e^{2} \mathrm{al}^{8}$ used $2 \%$ lignocaine, and in our study the commonest agent was $0.75 \%$ levobupivacaine in $77.5 \%$ of patients. It is possible that it could be related to the type of block, as in the study by Tan et al, ${ }^{7}$ patients either had a peribulbar or retrobulbar block, but Sugisaka et $a l^{8}$ used only retrobulbar blocks. In our study, a sub-Tenon's block 
was undertaken in $62(77.5 \%)$ patients with a peribulbar block in the remainder. We also found that patients having a sub-Tenon's as compared with a peribulbar block were more likely to see light during the procedure $(P=0.005)$. Unfortunately, Tan et $a l^{7}$ did not state how many of their patients had a peribulbar block, or whether it was associated with not perceiving light. It is difficult to explain how one specific type of block would be more likely to abolish light perception, particularly as differences have not been seen in studies on cataract surgery. ${ }^{2,4,6}$ Also, sub-Tenon's, peribulbar, and retrobulbar blocks have been shown to cause a similar level of disruption to optic nerve conduction immediately following administration. ${ }^{13}$

A number of studies on cataract surgery recommended that a detailed preoperative counselling should be mandatory, and should include comprehensive information about visual perceptions to relieve patients from unnecessary distress. ${ }^{1,2,5,14}$ Interestingly, in our study, only four patients wished to be forewarned about the visual sensations.

Overall, we found no statistical association between the visual perceptions and gender, preoperative visual acuity, preexisting ocular conditions, density of the block, or duration of surgery. Using logistic regression analysis, a number of significant associations were identified - younger people seeing flashing lights and instruments, patients having a sub-Tenon's block perceiving light, patients undergoing vitrectomy seeing more instruments, and those who did not receive any sedation seeing instruments. Care is needed to interpret these findings, as similar associations have not been reported previously. It is not surprising that the patients not having awake sedation perceived more visual perceptions, similarly the association with vitrectomy can be explained by the fact that vitrectomy involves the usage of greater number of instruments as compared with ROSO. However, the statistical associations with the other results are more difficult to explain.

Visual experiences are common during vitreoretinal surgery undertaken under LA, and not altered by the density of the block and not related to the preoperative visual acuity. Most people retain at least light perception, with many subjected to a wealth of other experiences, including colours, movements, and seeing instruments. Some patients found the visual sensations a pleasant experience with hardly any frightened by them.

Surprisingly, only a few patients wished to be informed about what they might experience during the surgery, Nevertheless, as healthcare providers, it is important that we are able to explain to patients what they might encounter during surgery, so those who do wish to be forewarned about these experiences can be reassured that they are not normally frightening.

\section{Acknowledgements}

We thank Roger Holder, Head of Statistics, Primary Care Clinical Sciences, University of Birmingham, for his valuable input into the statistical analysis of the data.

\section{References}

1 Prasad N, Kumar CM, Patil BB, Dowd TC. Subjective visual experience during phacoemulsification cataract surgery under sub-Tenon's block. Eye 2003; 17: 407-409.

2 Wickremasinghe SS, Tranos PG, Sinclair N, Andreou PS, Harris ML, Little BC. Visual perception during phacoemulsification cataract surgery under sub-Tenons anaesthesia. Eye 2003; 17: 501-505.

3 Rengaraj V, Radhakrishnan M, Au Eong KG, Saw SM, Srinivasan A, Mathew J et al. Visual experience during phacoemulsification under topical versus retrobulbar anesthesia: results of a prospective, randomized, controlled trial. Am J Ophthalmol 2004; 138: 782-787.

4 Chung CF, Lai JS, Lam DS. Visual sensation during phacoemulsification and intraocular lens implantation using topical and regional anesthesia. J Cataract Refract Surg 2004; 30: 444-448.

5 Ang CL, Au Eong KG, Lee SS, Chan SP, Tan CS. Patients' expectation and experience of visual sensations during phacoemulsification under topical anaesthesia. Eye 2007; 21: 1162-1167.

6 Tan CS, Au Eong KG, Kumar CM. Visual experiences during cataract surgery: what anaesthesia providers should know. Euro J Anaesthesiol 2005; 22: 413-419.

7 Tan CS, Mahmood U, O'Brien PD, Beatty S, Kwok AK, Lee VY et al. Visual experiences during vitreous surgery under regional anesthesia: a multicenter study. Am J Ophthalmol 2005; 140: 971-975.

8 Sugisaka E, Shinoda K, Ishida S, Imamura Y, Ozawa Y, Shinoda $\mathrm{H}$ et al. Patients' descriptions of visual sensations during pars plana vitrectomy under retrobulbar anesthesia. Am J Ophthalmol 2007; 144: 245-251.

9 Costen MT, Newsom RS, Wainwright AC, Luff AJ, Canning $\mathrm{CR}$. Expanding role of local anaesthesia in vitreoretinal surgery. Eye 2005; 19: 755-761.

10 Knight HM, Newsom RB, Canning CR, Luff AJ, Wainwright AC. Local anaesthesia for vitreoretinal surgery: an audit of patient and surgical experience. Eur J Ophthalmol 2001; 11: 366-371.

11 Brahma AK, Pemberton CJ, Ayeko M, Morgan LH. Single medial injection peribulbar anaesthesia using prilocaine. Anaesthesia 1994; 49: 1003-1005.

12 Kawaguchi N, Inoue M, Sugisaka E, Shinoda K, Tsubota K. Subjective visual sensation during vitrectomy under retrobulbar anesthesia. Am J Ophthalmol 2006; 141: 407-409.

13 Ramsay AS, Ray-Chaudhuri N, Dayan M, Walshaw D. Quantification of relative afferent pupillary defects induced by posterior sub-Tenon's, peribulbar, and retrobulbar anaesthetics. Br J Ophthalmol 2001; 85: 1445-1446.

14 Voon LW, Au Eong KG, Saw SM, Verma D, Laude A. Effect of preoperative counselling on patient fear from the visual experience during phacoemulsification under topical anesthesia: multicenter randomized clinical trial. J Cataract Refract Surg 2005; 31: 1966-1969. 